\title{
LA VEGETACIÓN DE LAS LAJAS EN LA REGIÓN DE LOMERÍO, SANTA CRUZ, BOLIVIA
}

\author{
Bonifacio MOSTACEDO; Marisol TOLEDO; Todd S. FREDERICKSEN
}

RESUMEN - Estudiamos la riqueza y composición de especies vegetales en tres afloramientos rocosos (lajas) en Lomerío, Santa Cruz, Bolivia, dentro de un bosque seco tropical. Determinamos la variación de la vegetación desde el centro hacia el borde de las lajas, además que caracterizamos algunas especies propias de este hábitat. La riqueza de especies varía entre lajas, pero, en forma conjunta esta riqueza puede ser mayor a otros tipos de bosque aledaños a las lajas. La riqueza de especies fue mayor en el borde que en el centro de las lajas. En el borde de las lajas, la riqueza de especies varía según la distancia al centro de las lajas; la mayor riqueza se encontró en los primeros $10 \mathrm{~m}$ del borde. En el centro de las lajas Deuterocohnia meziana Kuntze ex Mez fue la especie más dominante, mientras que las especies más abundantes fueron $D$. meziana y Monvillea kroenleinii R. Kiesling. En el borde de las lajas Anthurium plowmanii Croat, Pseudananas sagenarius (Arruda) Camargo, Urera baccifera L., Casearia gossypiosperma Briq., Aspidosperma rigidum Rusby y Cereus tacuaralensis Cárdenas fueron las especies más abundantes. De acuerdo a las clases diamétricas, se puede considerar que existe regeneración natural para las principales especies arbóreas. Las lajas son hábitats muy importantes que deben considerarse dentro del manejo forestal de bosques naturales adyacentes, puesto que constituyen lugares de alta diversidad y endemismo de plantas y animales y que son refugios y fuentes de alimentación de muchas especies de animales.

Palabras-clave: diversidad floristica, laja, inselberg, Bolivia, bosque tropical seco.

\section{Vegetation of Rock Outcrops in the Region of Lomerio, Santa Cruz, Bolivia}

ABSTRACT - Plant species richness and composition was studied on three rock outcrops (lajas) in a tropical dry forest in Lomerio, Bolivia. The variation in vegetation was determined from the center to the periphery of the influence of the rock outcrops and plant species indicators of rock outcrop habitats were identified. Species richness varied among lajas, but was higher in rock outcrop habitats compared to adjoining forests. Species richness was highest on the periphery of the rock outcrops compared to the vegetated islands on the center of the outcrop. The highest species richness was encountered $10 \mathrm{~m}$ from the vegetated border on the periphery of the pure rock surface. Deuterocohnia meziana Kuntze ex Mez was the most dominant (highest ground cover) plant species colonizing the center of the rock outcrops, while D. meziana and Monvillea kroenleinii R. Kiesling were the most abundant. On the periphery of the laja Anthurium plowmanii Croat, Pseudananas sagenarius (Arruda) Camargo, Urera baccifera L., Casearia gossypiosperma Briq., Aspidosperma rigidum Rusby and Cereus tacuaralensis Cárdenas were the most dominant species. Regeneration appears to be occurring for the principal tree species. Lajas are key habitats that should be considered in the natural management of forests in which they are imbedded. They constitute areas of high biodiversity and endemic species and are refuges and feeding areas for many animal species.

Key-Words: Plant diversity, inselberg, Bolivia, tropical dry forest, rock outcrop.

Proyecto BOLFOR, Casilla 6204, Santa Cruz, Bolivia Tel: 591-3-480766/480767, Fax: 591-3480854, bolfor@bibosi.scz.entelnet.bo 


\section{INTRODUCCIÓN}

En las tierras del Este de Bolivia se encuentra el escudo precámbrico que se extiende desde el Brasil. En estos lugares, caracterizado principalmente por tener suelos pobres, se desarrolla un mosaico de vegetación

conformado

principalmente del bosque semidecíduo pluviestacional, el bosque húmedo de llanura, el Cerrado y las lajas (Beck et al., 1993; Navarro, 1997). Las lajas son ambientes aislados ubicados dentro del bosque semideciduo pluviestacional o el bosque húmedo de llanura.

Las lajas también conocidas como domos o "inselbergs" son grandes rocas graníticas, sobresalientes y expuestas, de color negro, producto de la colonización de cianobacterias (Büdel et al., 1994). De igual forma, estos hábitats se caracterizan por tener suelos superficiales, ácidos, donde la materia orgánica va aumentando a medida que se aleja de las rocas expuestas.

Las lajas constituyen centros de diversidad y endemismo tanto de plantas y animales (Ibisch et al., 1995; Fredericksen et al., En Rev.). La alta diversidad de especies está dada por la yuxtaposición de distintos tipos de vegetación y por existencia de una gran variedad de microhábitats formados por la variación en la pendiente y profundidad del suelo. Por ello, las lajas fueron propuestas como reservas biológicas dentro de áreas de manejo forestal en los bosques secos (Fredericksen et al., en revisión).

Nuestro interés por estudiar estos hábitats resultó de la poca información cuantitativa de la vegetación. Algunos estudios, solo caracterizan cualitativamente la vegetación de las lajas (Killeen et al., 1990; Ibisch et al., 1995; Navarro, 1997) y no detallan la gran variación existente a nivel microambiental. Nuestra principal pregunta que tratamos de responder fue: ¿Cómo varía la vegetación del centro hacia el borde de las lajas? Nosotros observamos que las lajas tienen influencia en la vegetación más allá de lo que habitualmente se considera, y que esta influencia puede variar con la distancia, profundidad del suelo y la pendiente. El objetivo de este estudio fue determinar la diversidad y composición de las especies de plantas en relación a la distancia desde el centro de las lajas.

\section{METODOS}

\section{Sitios de Estudio}

El estudio lo realizamos en la región del Escudo Precámbrico de Lomerío, en Santa Cruz, Bolivia (16³2'58”S, 6150’29”W) (Fig. 1). La temperatura promedio en esta región es aproximadamente de 24.3 ${ }^{\circ} \mathrm{C}$, aunque encima de $10 \mathrm{~s}$ afloramientos rocosos puede llegar hasta los $50{ }^{\circ} \mathrm{C}$ (Nell Fredericksen, Com. Pers.). La precipitación anual oscila alrededor de los $1100 \mathrm{~mm}$; la zona presenta una temporada seca de 6-7 meses. Los suelos alrededor de las 
lajas son pobres y ácidos (entisoles), tienen poca profundidad, y son de origen granitico (Killeen \& Hinz, 1992). Esta región presenta un mosaico de vegetación formado principalmente por bosque semideciduo, Cerrado, y la vegetación alrededor de los afloramientos rocosos. Florística y estructuralmente, los afloramientos rocosos presentan una vegetación distinta a los bosques de sus alrededores (Ibisch et al., 1995, Killeen et al., 1990).

\section{Diseño de muestreo y recolección de datos}

Para cuantificar la vegetación seleccionamos tres lajas de distinto tamaño y forma. Dentro de cada laja consideramos dos hábitats distintos, el primer hábitat (centro) estuvo situado en los mismos afloramientos de roca, y el segundo hábitat (borde) estuvo situado alrededor de cada afloramiento (Fig. 2). Desde un punto central de cada laja trazamos seis líneas en diferentes direcciones, en las cuales muestreamos la vegetación. Nosotros hemos considerado que tanto el centro como el borde están influenciados por la presencia de roca granítica, y que estos microhábitats que conforman una laja están rodeados por otros tipos de vegetación denominadas bosque semideciduo pluviestacional y bosque ribereño (Navarro, 1997).

La vegetación del centro de las lajas muestreamos en aquellas islas interceptadas por la linea de muestreo. $\mathrm{En}$ islas pequeñas hasta $5 \mathrm{~m}^{2}$ muestreamos un cuadro de $1 \mathrm{~m}^{2}$, mientras que en islas mayores a $5 \mathrm{~m}^{2}$ muestreamos entre 2 y $3 \mathrm{~m}^{2}$. Asimismo, la vegetación alrededor de los afloramientos rocosos (borde) hicimos el muestreo en las líneas anteriormente establecidas. Cada 10 $\mathrm{m}$ hasta los $50 \mathrm{~m}$ evaluamos las plantas menores a $2 \mathrm{~m}$ de altura en una superficie de $1 \mathrm{~m}^{2}$, las plantas mayores a $2 \mathrm{~m}$ de altura y menores a $10 \mathrm{~cm}$ DAP en áreas de $4 \mathrm{~m}^{2}$, y las plantas mayores a $10 \mathrm{~cm}$ de DAP en áreas de $100 \mathrm{~m}^{2}$. En las plantas del centro de cada laja tomamos datos de la cobertura y abundancia de especies, mientras que en las plantas del borde de las lajas consideramos datos de la abundancia, DAP, y altura. La cobertura de las plantas medimos evaluando el porcentaje de la proyección vertical sobre el suelo; esta proyección fue medida dentro de cada cuadrante, en porcentaje con intervalos de $10 \%$. La abundancia se midió en terminos de número de individuos existentes en cada cuadrante de muestreo.

\section{Análisis de datos}

Para determinar la variación florística entre lajas obtuvimos la riqueza de familias y especies totales de cada laja. Para determinar la similitud de especies entre lajas utilizamos el Indice de Sorensen (Magurran, 1988). Mediante un análisis de agrupación (Euclidean Cluster Analisis) (McCune\&Mefford, 1997) examinamos el grado de similitud florística existente en el gradiente desde el centro y el extremo 


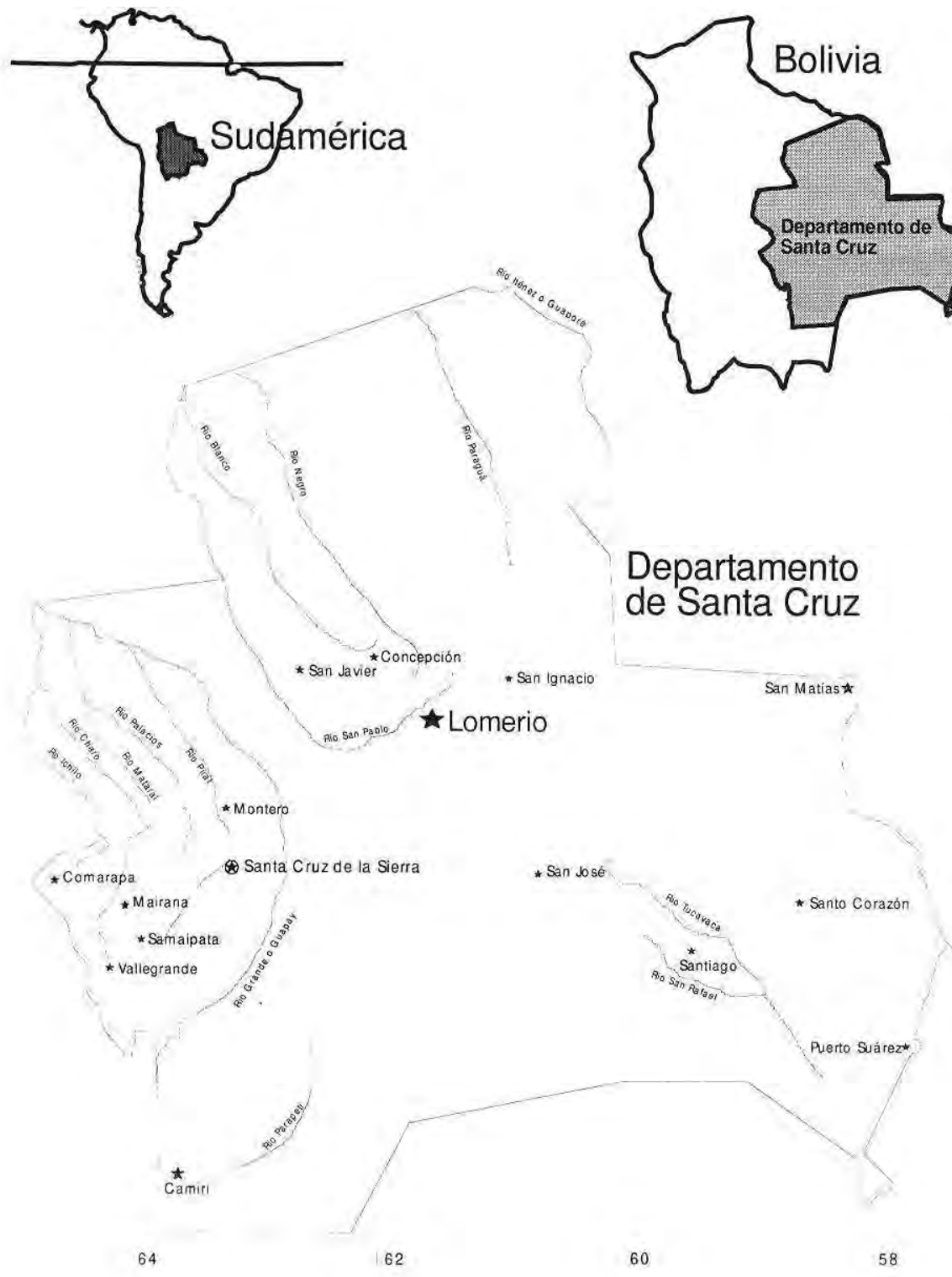

Figura 1. Ubicación de la zona de estudio en la región de Lomerio, Santa Cruz, Bolivia 


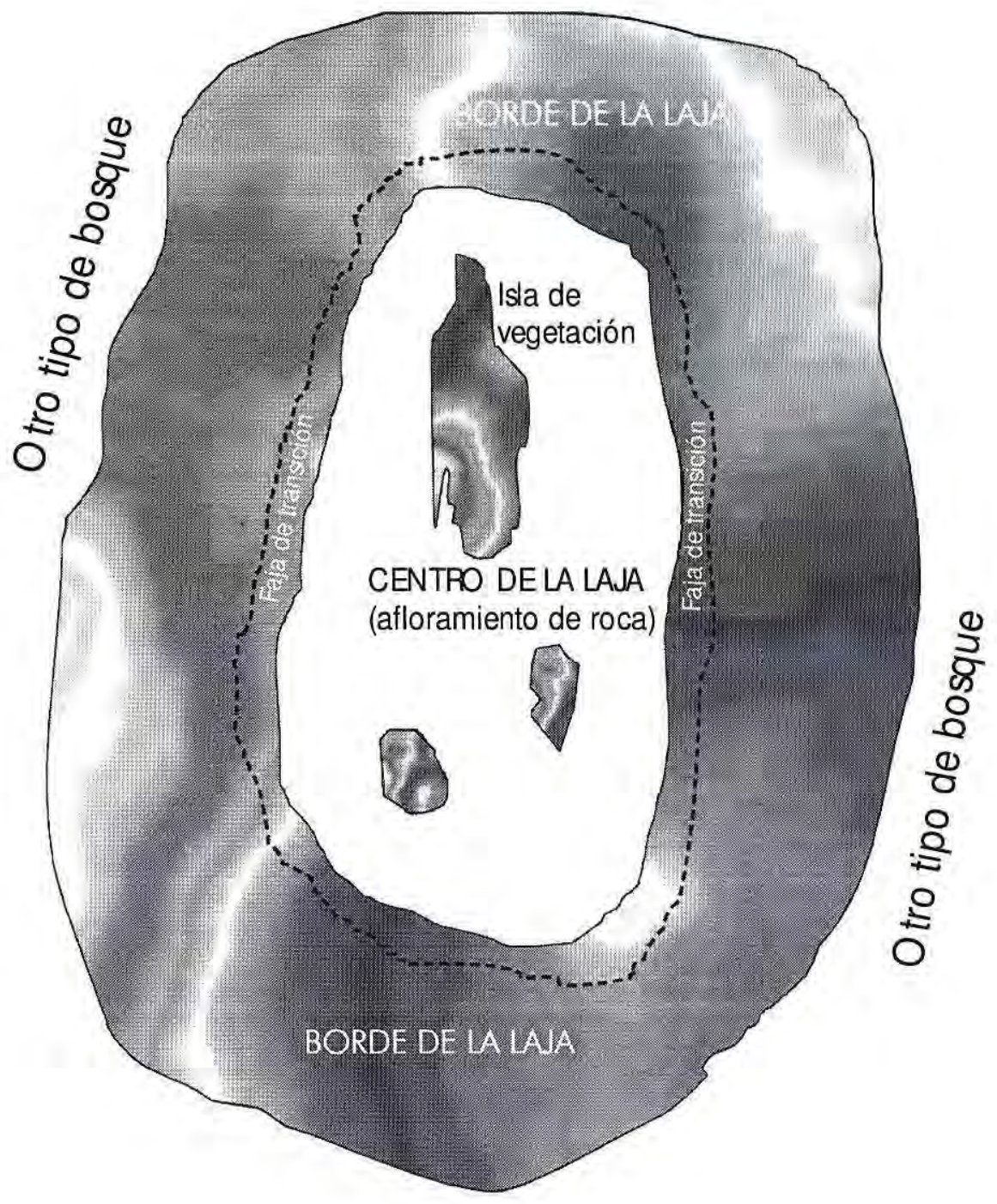

Figura 2. Croquis general de una laja y su influencia, indicando los principales microhábitats. 
de las lajas, tomando en cuenta las especies y la frecuencia de cada especie. Para las plantas del centro de las lajas también determinamos la riqueza, dominancia y abundancia, y para las del borde la riqueza, frecuencia y abundancia de las especies.

\section{RESULTADOS}

\section{Riqueza, Frecuencia y Similaridad de Especies}

En la vegetación de las lajas las familias con mayor riqueza de especies fueron las Bignoniaceae (42 spp.), Leguminosae (36 spp.), Sapindaceae (13 spp.) y las Pteridophytas (13 spp.). En las tres lajas estudiadas encontramos 310 especies de plantas, incluyendo las Pteridophytas, Dicotiledoneas y Monocotiledoneas. En una de las lajas encontramos mayor número de especies respecto a las otras dos restantes (Fig. 3).

En el centro de las lajas la riqueza de familias y especies vario enormemente (Tab. 1). Asimismo, la similaridad de especies en el centro de las lajas varío de 8 a $24 \%$. En las plantas del borde de las lajas, la riqueza de familias y especies fue muy variable (Tab. 1). En plantas menores a $2 \mathrm{~m}$ de altura, la similaridad de especies osciló entre 23 y $36 \%$. En cambio en plantas mayores a $2 \mathrm{~m}$ de

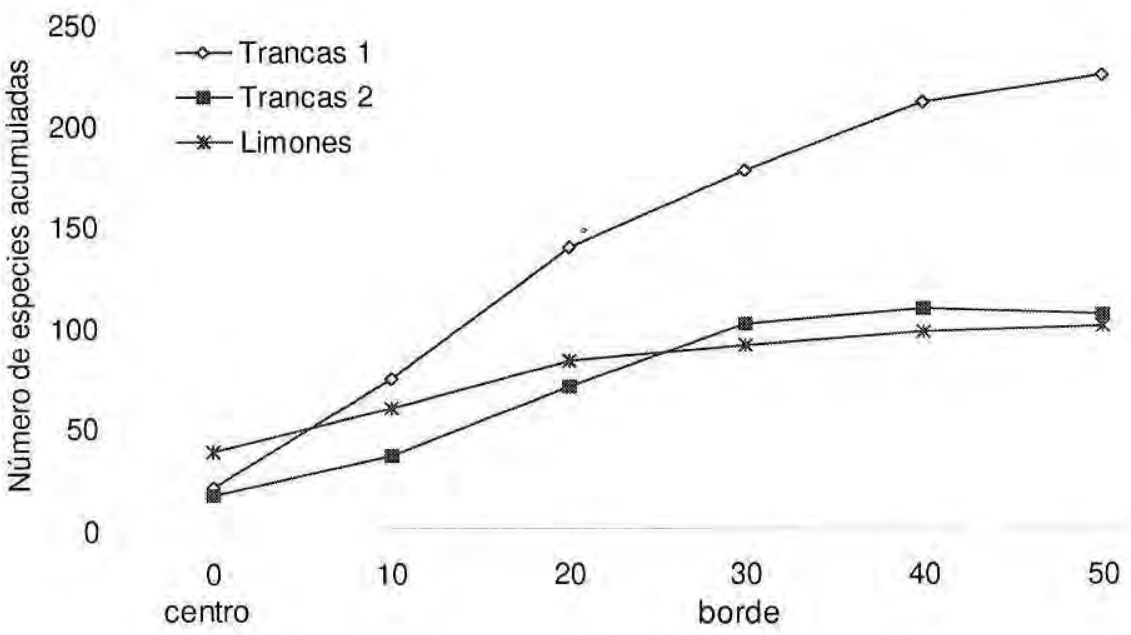

Distancia (m)

Figura 3. Curva de especies acumuladas en relación a la distancia desde el centro al borde de las lajas. 
altura y menores a $10 \mathrm{~cm}$ de DAP la similaridad de especies se redujo entre 14 y $24 \%$. En plantas con DAP mayores a $10 \mathrm{~cm}$ las lajas fueron más parecidas en su flora $(40-58 \%$ de similaridad).

En el gradiente del centro al extremo de la laja se evidenció una variación de la riqueza y frecuencia de las especies. Las plantas por debajo de los dos metros de altura del centro de la laja tienen alguna similitud con los primeros $10 \mathrm{~m}$ del borde de la laja; también se pudo constatar que entre los 20 y 50 metros de distancia del borde existe más similitud entre las especies (Fig. 4a). En el borde de las lajas, a medida que se aleja del centro de la laja, las especies pueden agruparse en tres grupos (Figs. 4b, 4c).

En las tres categorias de plantas la frecuencia de especies parece ser diferente en los 10 primeros metros del centro al borde de la laja. Entre las especies menores a dos metros, $A n$ thurium plowmanii Croat, Deuterocohnia meziana Kuntze ex Mez, Monvillea kroenleinii R. Kiesling y Cochlospermun vitifolium (Willd.) Willd. ex Spreng. fueron las especies más frecuentes de los primeros $10 \mathrm{~m}$ del borde. Entre las especies mayores a $2 \mathrm{~m}$ de altura y menores a $10 \mathrm{~cm}$ DAP, Bauhinia rufa (Bong.) Steud., Cereus tacuaralensis Cárdenas y Cochlospermun vitifolium fueron las más frecuentes. Por su parte, los árboles (con más de $10 \mathrm{~cm}$ de DAP) más frecuentes fueron Cereus tacuaralensis, Aspidosperma rigidum Rusby, Pseudobombax marginatum (A. St.-Hil., Juss \& Cambess.) A. Robyns y Callisthene fasciculata Mart.

\section{Dominancia y Abundancia de Especies}

En el centro de las lajas, el área cubierta por vegetación fue entre 10 a $20 \%$. Dentro de las áreas cubiertas

Tabla 1- Riqueza de familias y especies de las plantas de Las Lajas.

\begin{tabular}{|c|c|c|c|c|c|c|c|c|}
\hline & \multicolumn{2}{|c|}{ CENTRO LAJA } & \multicolumn{6}{|c|}{ BORDE LAJA } \\
\hline & \multicolumn{2}{|c|}{$\begin{array}{c}\text { Plantas }<2 \mathrm{~m} \text { de } \\
\text { altura }\end{array}$} & \multicolumn{2}{|c|}{$\begin{array}{c}\text { Plantas }<2 \mathrm{~m} \text { de } \\
\text { altura }\end{array}$} & \multicolumn{2}{|c|}{$\begin{array}{c}\text { Plantas }>2 \mathrm{~m} \mathrm{y}< \\
10 \mathrm{~cm} \text { DAP }\end{array}$} & \multicolumn{2}{|c|}{$\begin{array}{c}\text { Plantas }>10 \mathrm{~cm} \\
\text { de altura }\end{array}$} \\
\hline & Familia & Especie & Familia & Especie & Familia & Espe cie & Familia & Especie \\
\hline Laja 1 & 11 & 20 & 39 & 129 & 26 & 73 & 20 & 41 \\
\hline Laja 2 & 11 & 12 & 36 & 79 & 21 & 37 & 20 & 39 \\
\hline Laja 3 & 19 & 38 & 42 & 75 & 21 & 26 & 12 & 19 \\
\hline Promedio & 13.6 & 23.3 & 29.2 & 94.3 & 17 & 45.3 & 13 & 33.0 \\
\hline
\end{tabular}



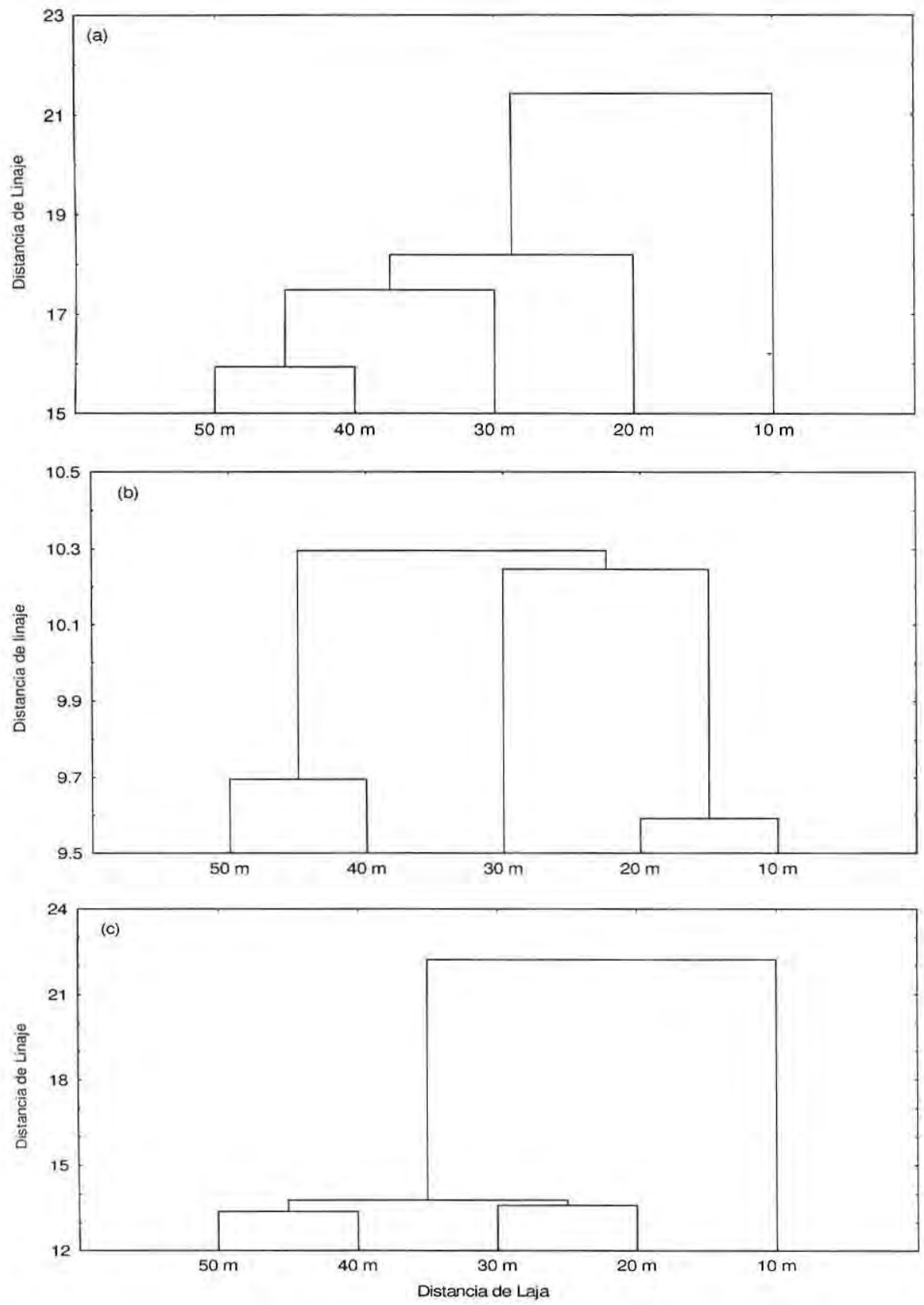

Figura 4 - Dendrograma de la similaridad de especies alrededor (borde) de las lajas por el método Euclidean Cluster Análisis. Los dendrogramas se hicieron tomando en cuenta las especies y la frecuencia de especies a diferentes distancias de las lajas. (a) Plantas menores a dos metros de altura, (b) plantas mayores a 2 metros de altura y menores a $10 \mathrm{~cm}$ de DAP, y (c) Plantas mayores a $10 \mathrm{~cm}$ de DAP. 
por vegetación, la mayor superficie estuvo cubierta por plantas vasculares (50-70\%), mientras que los musgos cubren alrededor del 30\%. Dentro de las plantas vasculares la especie más dominante y abundante casi en todas las lajas estudiadas fue Deuterocohnia meziana (Tab. 2).

En el borde de las lajas, la abundancia de cada especie varió según la laja. Entre las plantas menores a 2 metros, Anthurium plowmanii fue la más abundante, aunque Pseudananas sagenarius (Arruda) Camargo, Malpighiaceae sp. 1, Peperomia pachydermis C. DC. y Aspidosperma rigidum también son importantes en su abundancia (Tab. 3). En las plantas mayores a dos metros y menores a $10 \mathrm{~cm}$ de DAP,

Tabla 2 - Dominancia ( $\%$ de cobertura) y abundancia relativa (ABR) de las principales especies en el centro de las lajas.

\begin{tabular}{lcc}
\hline Especies & Cobertura (\%) & ABR (\%) \\
\hline Laja 1 & & \\
Deutercohnia meziana Kuntze ex Mez & 62.0 & 57.0 \\
Helecho sp. 2 & 15.5 & 13.0 \\
Mimosa neptunioides Harms & 12.7 & 14.0 \\
Bignoniaceae sp. 6 & 1.5 & 0.4 \\
Helecho sp. 4 & 1.6 & 8.1 \\
Sebastiania sp. & 2.5 & 5.4 \\
& & \\
Laja 2 & & \\
Melastomataceae sp. 1 & 33.0 & 2.5 \\
Deuterocohnia meziana & 15.0 & 25.0 \\
Monvillea kroenleinii & 11.8 & 32.5 \\
Bignoniaceae sp. 26 & 10.0 & 2.5 \\
Ananas ananassoides (Baker) L. B. Sm. & 8.3 & 17.5 \\
& & \\
Laja 3 & & \\
Deuterocohnia meziana & 100.0 & 63.9 \\
Norantea guianensis & 14.2 & 2.6 \\
Doryopteris pedata (L.) Fée & 14.2 & 1.4 \\
Mimosa xanthocentra Mart. & 11.6 & 1.1 \\
Graminae sp. 4 & 9.0 & 1.4 \\
\hline
\end{tabular}


Urera baccifera (L.) Gaudich. ex Wedd., Casearia gossypiosperma Briq., Eugenia sp. 1 y Cochlospermun vitifolium fueron las especies más abundantes, aunque en distintas lajas. En plantas con DAP mayor a $10 \mathrm{~cm}$, en todas las lajas, Cereus tacuaralensis y Aspidosperma rigidum fueron las más abundantes (Tab. 3).

\section{Especies características}

Las lajas son un tipo de hábitat donde a parte de que se mezclan especies de distintos tipos de hábitat, tambien son lugares donde se desarrollan especies propias o caracteristicas. En el centro de las lajas, algunas especies como Deuterocohnia meziana (Bromeliaceae), Anthurium plowmanii (Araceae), Monvillea kroenleinii R. Kiesling (Cactaceae), Tillandsia didisticha (E. Morren) Baker (Bromeliaceae), Norantea guianensis Aubl. (Macgraviaceae), Dioscorea sp. (Dioscoreaceae) y Heteranthera limosa (Sw.) Willd. (Pontederiaceae, acuática) pueden ser consideradas como especies herbaceas y arbustivas características. En la vegetación del borde de las lajas, Commiphora leptophloeos (Mart.) J. B. Gillet (Burseraceae, árbol), Esenbeckia almawillia Kaastra (Rutaceae, arbusto), Echinopsis hammerschmidii Cárdenas (Cactaceae, arbusto) y Ficus calyptroceras (Miq.) Miq. (Moraceae, arbol), son consideradas como las principales especies características.

Formas de vida, clases diamétricas y de altura
En el centro de las lajas las hierbas, arbustos y trepadoras presentaron la mayor riqueza de especies (Tab. 4). Algunos árboles pequeños de Tabebuia impetiginosa (Mart. ex DC.) Standl. y Cochlospermun vitifolium pueden encontrarse en las islas del centro de las lajas. En el borde de las lajas, el grupo de especies con mayor número de especies por debajo de los dos metros fueron las trepadoras, seguidas de los arbustos y hierbas. Dentro de las plantas mayores a dos metros y menores a $10 \mathrm{~cm}$ de DAP, la riqueza de especies fue más alta en los arbustos y trepadores. Todas las plantas mayores a $10 \mathrm{~cm}$ de DAP fueron árboles. Los helechos, se encontraron más en el borde que en el centro de las lajas.

Entre los árboles mayores a 10 $\mathrm{cm}$ de DAP situados en el borde de las lajas, el promedio general de DAP fue de $20 \mathrm{~cm}$; el $62 \%$ de los individuos tuvieron diámetros por debajo del promedio general. Los árboles más gruesos fueron Ficus calyptoceras, Platymiscium ulei Harms ex Harms y Machaerium scleroxylon Tul. Las especies más abundantes tuvieron una distribución diamétrica exponencial negativa (J invertida), a excepción de $F$. calyptoceras que tiene una distribución regular en todas las clases diamétricas (Tab. 5). Los árboles en las lajas generalmente fueron más bajos que el bosque aledaño, estos pueden llegar hasta los $20 \mathrm{~m}$ de altura. En promedio, los árboles más altos fueron Schinopsis brasiliensis Engl. (16 m), Copaifera chodatiana Hassl. 
Tabla 3 - Especies más abundantes (abundancia relativa) en el borde de tres lajas distintas de la región de Lomerío.

\begin{tabular}{|c|c|c|c|c|}
\hline Especies & Familias & Laja 1 & Laja 2 & Laja 3 \\
\hline \multicolumn{5}{|l|}{ Plantas menores a $2 \mathrm{~m}$ de altura } \\
\hline Anthurium plowmanii Croat & Araceae & 10.1 & 3.5 & 6.4 \\
\hline Pseudananas sagenarius (Arruda) Camargo & Bromeliaceae & 2.5 & 5.7 & 3.2 \\
\hline Peperomia pachydermis C. DC. & Piperaceae & 2.8 & 2.1 & 2.6 \\
\hline Aspidosperma rigidum Rusby & Apocynaceae & 1.5 & 2.8 & 2,4 \\
\hline Serjania sp. 1 & Sapindaceae & 4.9 & 0.7 & 2.3 \\
\hline Lasiacis sorghoidea (Desv.) Hitchc. \& Chase & Graminae & 2.5 & 3.2 & 2.2 \\
\hline Malpighiaceae sp. 1 & Malpighiaceae & 0.3 & 5.3 & 2.2 \\
\hline Eugenia sp. 1 & Myrtaceae & 2.5 & 2,1 & 2.2 \\
\hline Deuterocohnia meziana & Bromeliaceae & 1.2 & 0.4 & 2.0 \\
\hline Desconocida & Desconocida & 4.9 & 0.0 & 2.0 \\
\hline Serjania marginata Casar. & Sapindaceae & 0.0 & 3.9 & 1.9 \\
\hline Monvillea kroenleinii R. Kiesling & Cactaceae & 1.2 & 3.2 & 1.8 \\
\hline Otras & & 63.6 & 65.1 & 66.8 \\
\hline \multicolumn{5}{|l|}{ Plantas mayores a $2 \mathrm{~m}$ de altura $\mathrm{y}$} \\
\hline menores a $10 \mathrm{~cm}$ de DAP & Urticaceae & 6.4 & 1.1 & 3.3 \\
\hline Urera baccifera L. & Flacourtiaceae & 2.4 & 5.6 & 3.3 \\
\hline Casearia gossypiosperma Briq. & Myrtaceae & 4.0 & 5.6 & 0.0 \\
\hline Eugenia sp. 1 & Myrtaceae & 3.2 & 4.5 & 0.0 \\
\hline Aspidosperma rigidum Rusby & Bixaceae & 1.6 & 1.1 & 8.3 \\
\hline Cochlospermun vitifolium (Willd.) Willd. ex Spreng. & Leguminosae & 2.4 & 3.4 & 0.0 \\
\hline Anadenanthera macrocarpa (Benth.) Brenan & Trigoniaceae & 4.0 & 1.1 & 0.0 \\
\hline Trigonia boliviana Warm. & Bignoniaceae & 0.0 & 4.5 & 0.0 \\
\hline Arrabidaea fagoides Burreau & Cactaceae & 0.8 & 1.1 & 3.3 \\
\hline Cereus tacuaralensis Cárdenas & Flacourtiaceae & 0.8 & 3.4 & 0.0 \\
\hline Casearia arborea (Rich.) Urb. & Graminae & 3.2 & 0.0 & 0.0 \\
\hline Chusquea ramosissima Pilg. & Leguminosae & 0.0 & 0.0 & 6.7 \\
\hline Mimosa xanthocentra Mart. & & 68.8 & 61.8 & 65.0 \\
\hline
\end{tabular}

\section{Arboles mayores a $10 \mathrm{~cm}$ de DAP}

Cereus tacuaralensisn Cárdenas

Aspidosperma rigidum

Astronium urundeuva (Allemao) Engl.

Tabebuia impetiginosa (Mart. ex DC.) Standl.

Eriotheca roseorum (Cuatrec.) A. Robyns

Ficus eximia Schott ex Spreng.

Pseudobombax marginatum (Martius \& Zuccarini) A. Robyns

Sterculia apetala (Jacq.) H. Karst.

Ficus calyptroceras (Miq.) Miq.

Aspidosperma cylindrocarpon Müll. Arg.

Anadenanthera macrocarpa

Centrolobium microchaete (Mart, ex Benth.) Lima ex G. P. Lewis

Otras

\begin{tabular}{llll} 
Cactaceae & 16.0 & 11.9 & 19.0 \\
Apocynaceae & 13.8 & 14.1 & 11.1 \\
Anacardiaceae & 7.2 & 3.7 & 4.8 \\
Bignoniaceae & 6.6 & 1.5 & 3.2 \\
Bombacaceae & 5.0 & 3.7 & 3.2 \\
Moraceae & 5.0 & 0.0 & 0.0 \\
Bombacaceae & 3.9 & 5.2 & 3.2 \\
Sterculiaceae & 3.9 & 3.7 & 0.0 \\
Moraceae & 3.3 & 3.0 & 1.6 \\
Apocynaceae & 2.8 & 3.7 & 0.0 \\
Leguminosae & 2.2 & 6.7 & 4.8 \\
Leguminosae & 1.1 & 8.9 & 0.0 \\
& 29.2 & 33.9 & 49.1 \\
\hline
\end{tabular}


$(15.5 \mathrm{~m})$, Platymiscium ulei $(15 \mathrm{~m})$, Machaerium scleroxylon $(13 \mathrm{~m})$, Hymenaea courbaril L. (12 m) y Pseudobombax longiflorum (Martius \& Zuccarini) A. Robyns (12 m). microgeomorfologia que puede existir, por el tamaño de cada laja, y/o por el número de islas de vegetación formadas en el centro de las lajas.

Los datos indican que con los

Tabla 4 - Riqueza de especies según formas de vida y categorias de tamaño en el centro y borde de las lajas.

\begin{tabular}{|c|c|c|c|c|}
\hline \multirow[b]{2}{*}{ Formas de vida } & \multirow{2}{*}{$\frac{\text { CENTRO LAJA }}{<2 \mathrm{~m} \text { altura }}$} & \multicolumn{3}{|c|}{ BORDE LAJA } \\
\hline & & $<2 \mathrm{~m}$ altura & $\begin{array}{c}>2 \mathrm{~m}<10 \\
\mathrm{~cm} \mathrm{DAP}\end{array}$ & $>10 \mathrm{~cm} \mathrm{DAP}$ \\
\hline Arboles & 6 & 27 & 28 & 50 \\
\hline Arbustos & 14 & 47 & 36 & 0 \\
\hline Bromelias & 4 & 5 & 0 & 0 \\
\hline Epifitas & 0 & 3 & 3 & 0 \\
\hline Hierbas & 16 & 40 & 11 & 0 \\
\hline Musgos & 2 & 2 & 0 & 0 \\
\hline Helechos & 5 & 15 & 0 & 0 \\
\hline Trepadoras & 13 & 76 & 31 & 0 \\
\hline
\end{tabular}

\section{DISCUSIÓN}

Los afloramientos rocosos (lajas) son hábitats aislados $\mathrm{y}$ distintos en su riqueza de especies. La temperatura, el tipo de roca, el tamaño de las lajas (Porembski et al., 1996) y la profundidad del suelo puede estar determinando el tipo de vegetación caracteristica. Ibisch et al. (1995) mencionan que la composición de especies en cada laja puede estar influenciada también por la especiación, extinción, colonización y aislamiento. A nivel microambiental, es probable que las lajas esten influenciadas por el número de microhábitats debido a la muestreos se obtuvieron la mayor cantidad de especies, incluso mayor a la encontrada por otros autores (Killeen \& Nee, 1991; Killeen et al., 1998). Es probable que los otros estudios hayan considerado a las lajas solamente al centro y la transición entre el centro y borde de toda la influencia de la laja considerada en este estudio. La influencia de las lajas es más notable en aquellas ubicadas dentro de los bosques húmedos por sus caracteristicas de hábito y fenologia de las especies que habitan en estas (Ibisch et al., 1995). También, se observa que la riqueza de especies en la vegetación de las lajas es mayor que en las bajuras y llanuras del bosque 
Tabla 5 - Abundancia de las principales especies arbóreas de los alrededores de las lajas según clases diamétricas.

\begin{tabular}{lccccc}
\hline Especies & $10-20 \mathrm{~cm}$ & $20-30 \mathrm{~cm}$ & $30-40 \mathrm{~cm}$ & $40-50 \mathrm{~cm}$ & $>50 \mathrm{~cm}$ \\
\hline Anadenanthera macrocarpa & 6 & 4 & 5 & 1 & 0 \\
Aspidosperma rigidum & 43 & 6 & 2 & 0 & 0 \\
Astronium urundeuva & 12 & 7 & 2 & 0 & 0 \\
Centrolobium microchaete & 6 & 7 & 1 & 0 & 0 \\
Cereus tacuaralensis & 51 & 5 & 0 & 1 & 0 \\
Ficus calyptoceras & 3 & 1 & 2 & 3 & 2 \\
Pseudobombax marginatum & 7 & 7 & 1 & 0 & 1 \\
Tabebuia impetiginosa & 8 & 4 & 3 & 0 & 1 \\
\hline
\end{tabular}

semideciduo pluviestacional (Killeen et al., 1998; Navarro, 1997). A pesar de ello, la diversidad parece ser más baja en comparación con las lajas de Venezuela y Brasil (Cody, 1968).

Las lajas tienen especies de distintos tipos de bosque. Un grupo comparte con el bosque semideciduo chiquitano, tales como Aspidosperma rigidum, Acosmiun cardenasii $\mathrm{H}$. S. Irwin \& Arroyo, Aspidosperma macrocarpon Mart., Caesalpinia pluviosa DC., Astronium urundeuva (Allemao) Engl., Platymiscium ulei, Pseudoananas sagenarius, Sterculia apetala (Jacq.) H. Karst. y Tabebuia impetiginosa (Navarro, 1997). Otro grupo de plantas, comparte con el Cerrado (sensu lato), tales como Cochlospermun vitifolum, Pseudobombax marginatum, $P$. longiflorum, Callisthene fasciculata, Athyana weinmannifolia (Griseb.) Radlk., Bauhinia rufa y Agonandra sp. La combinación de especies de distintos hábitats y la influencia de diferentes factores ambientales, tales como material parental, temperatura del sustrato, profundidad y calidad del suelo, hacen que la vegetación de las lajas tenga una composición y fisonomía distinta (Killeen et al., 1990).

A pesar que en este artículo mencionamos algunas especies características de las lajas, las tres lajas analizadas no representan a todas las especies propias que existen en todas las lajas de la región del escudo precámbrico. Vellozia tubiflora (A. Rich.) Kunth, Frailea chiquitana Cárdenas, Gymnocalycium chiquitanum Cárdenas y Thrasya crucensis Killeen, también son algunas de las especies propias de las lajas (Ibisch et al., 1995; Navarro, 1997).

La vegetación de las lajas varía del centro hacia el extremo de la laja. En términos generales, la riqueza de especies en la transición entre el centro y el borde de las lajas, y a 
medida que se aleja del centro de la laja, el número de especies va reduciendo. Similar resultado se encontró en afloramientos rocosos de Australia (Freeland et al., 1988). Es probable que el factor determinante en los cambios de especies y frecuencia sea la profundidad del suelo y la pendiente. En áreas con suelos superficiales la especies predominantes son herbáceas y arbustivas, además estas son resistentes a las altas temperaturas que hay en los afloramientos rocosos. En sitios con altas pendientes la riqueza de especies es más baja y cambia bruzcamente de un lugar a otro. Asimismo, en la franja que divide el centro y el borde de la lajas la diversidad de plantas aumenta, debido a las condiciones microambientales que se genera en estos sitios, las que son óptimas para una gran variedad de especies. El borde de las lajas está más influenciado por la presencia de especies de la vegetación aledaña.

Parece ser que las lajas son lugares favorables para la regeneración natural de un grupo importante de especies arbóreas que se encuentran en las llanuras del bosque semideciduo pluviestacional. Inclusive, algunas especies como Cedrela fissilis Vell., Aspidosperma rigidum, Sterculia apetala, Pseudobombax marginatum y Astronium urundeuva tienen mejor regeneración que en otros tipos de bosque. Individuos adultos, aunque no plántulas, de Amburana cearensis (Allemao) A. C. Sm. son más abundantes en la transición entre el centro y el borde de las lajas.
Las lajas constituyen hábitats muy diversos con una considerable cantidad de especies endémicas tanto de plantas como de animales (Ibisch et al., 1995; Fredericksen et al., En Rev.). Los afloramientos graníticos por ser fuentes de alimentos, agua y algunas condiciones microclimáticas, permiten una mayor riqueza y abundancia de animales (Fredericksen et al., En Rev.). Asimismo, las lajas pueden ser incluídas dentro de la planificación del manejo forestal de los bosques secos tropicales, dándole utilidad como fuentes de árboles semilleros o ambientes con fines de mantener la biodiversidad.

\section{AGRADECIMIENTOS}

Este estudio fue financiado por el Proyecto de Manejo Forestal Sostenible de los Bosques de Bolivia (BOLFOR). Agradecemos por la ayuda en el campo a Nell Fredericksen y Marcela Pereira.

\section{Bibliografia citada}

Beck, S. G.; Killeen, T. J.; García E. E. 1993. Vegetación. Ev: T. J. Killeen, S. J. Beck y E, García (Eds.). Guia de Arboles de Bolivia. Herbario Nacional de Bolivia y Missouri Botanical Garden. La Paz, Bolivia. p. 6-23.

Büdel, B.; Lüttge, U.; Stelzer, R.; Huber, O.; Medina, E. 1994. Cyanobacteria of rocks and soils of the Orinoco Lowlands and the Guayana Uplands, Venezuela. Bot. Acta, 107: $422-431$.

Cody, M. L, 1968. Diversity, rarity, and conservation in Mediterranean-climate regions. En: Soulé, M. (Ed.). Conservation biology: the science of scarcity and diversity. Sinauer Associates Inc., Sunderland Massachusetts. p. 123-152. 
Fredericksen, N. J.; Fredericksen, T. S.; Flores, B.; McDonald, E.; Rumíz, D. En Revisión. Importance of granitic rock outcrops to vertebrate species in a Bolivian tropical forest. Journal of Biogeography.

Freeland, W. J.; Winter, J. W.; Raskin, S. 1988. Australian rock-mammals: A phenomenon of the seasonally dry tropics. Biotropica, 20(1): 70-79.

Ibisch, P. L.; Rauer, G.; Rudolph, D.; Barthlott, W. 1995. Floristic, biogeographical, and vegetational aspects of Pre-Cambrian rock outcrops (inselbergs) in eastern Bolivia. Flora, 190: 299-314.

Killeen, T. J.; Hinz, P. N. 1992. Grasses of the Precambrian Shield region in eastern lowland Bolivia, I. Habitat preferences. Journal of Tropical Ecology, 8: 389-407.

Killeen, T. J.; Jardim,A,; Mamani, F,; Rojas, N. 1998. Diversity, composition, and structure of a tropical deciduous forest in the Chiquitania region of Santa Cruz, Bolivia. Journal of Tropical Ecology, 14; 803-827.

Killeen, T. J.; Louman, B. T.; Grimnwood, T. 1990. La Ecología paisajística de la región de Concepción y Lomerio en la provincia Ñuflo de Chavez, Santa Cruz. Ecologia en Bolivia, 16: 1-45.
Killeen, T. J.; Nee, M. 1991. Catálogo de las plantas sabaneras de Concepcion, Depto. Santa Cruz, Bolivia. Ecologia en Bolivia, 16: 53-71.

Magurran, E. A. 1988. Ecological Diversity and its Measurement. Princeton University Press. $179 \mathrm{p}$.

McCune, B. \& M. J. Mefford. 1997. Multivariate Analysis of Ecological Data. PCORD for Windows, MjM Software, Gleneden Beach, Oregon, U.S.A.

Navarro, G. 1997. Contribución a la clasificación ecológica y floristica de los bosques de Bolivia. Revista Boliviana de Ecologia y Conservación Ambiental, 2: 337.

Porembski, S.; Szarzynski, J.; Mund, J.; Barthlott, W. 1996. Biodiversity and vegetation of small-sized inselbergs in a West African rain forest (Tai, Ivory Coast). Journal of Biogeography, 23:47-55. 